\title{
ALLOTMENT OF AIRCRAFT SPARE PARTS USING GENETIC ALGORITHMS
}

\author{
Pascale Batchoun \\ Département d'informatique et de recherche \\ opérationnelle \\ Université de Montréal \\ Montréal QC - Canada \\ Jacques A. Ferland * \\ Département d'informatique et de recherche \\ opérationnelle \\ Université de Montréal \\ Montréal QC - Canada \\ ferland@iro.umontreal.ca

\section{Robert Cléroux} \\ Département de mathématiques et de statistique \\ Université de Montréal \\ Montréal QC - Canada \\ * Corresponding author/autor para quem as correspondências devem ser encaminhadas \\ Recebido em 04/2002, aceito em 11/2002 após 1 revisão
}

\begin{abstract}
In this paper we attempt to determine the optimal allocation of aircraft parts used as spares for replacement of defective parts on-board of a departing flight. In order to minimize the cost of delay caused by unexpected failure, Genetic algorithms (GAs) are used to allocate the initial quantity of parts among the airports. GAs are a class of adaptive search procedures, that distinguish themselves from other optimization techniques by the use of concepts from population genetics to guide the search. Problem-specific knowledge is incorporated into the problem and efficient parameters are identified and tested for the task of optimizing the allocation of parts. The approach is illustrated by numerical results.
\end{abstract}

Keywords: genetic algorithms; allotment of spares; cost of delay. 


\section{Introduction}

When a repairable item on an aircraft becomes defective, it is removed and replaced by another item from the spare stock. The defective part is then sent to the maintenance shop for repair. Should the station not have a spare part in stock, the aircraft will remain on ground and will be delayed until an incoming flight brings a replacement part from the maintenance shop or from a neighboring station. In this context, a repairable item represents any aircraft part from a small electronic component to a whole engine. Spare inventory is placed at line stations to decrease time delays due to unanticipated failures, and is placed also at the maintenance shop to quickly replenish remote station allotments while their parts are being repaired.

An agreement ("International Airlines Technical Pool") between airlines exists to share certain parts at specific stations when parts fail. An airline is said to be the provider of a part at a specific station, when all the participant airlines of the agreement can borrow from the spare stock of the provider. In this case, a minimum of one part is allocated by the provider airline at that station.

A station is said to have maintenance ability for a certain part, when there is maintenance staff at the station accredited to remove and replace the defective part on the aircraft. When there is no maintenance ability for a specific part at a station, the aircraft with a defective part remains on ground until a technician flies into the station along with a replacement part. Therefore, the stations are given nil allotment for parts with no maintenance ability.

In this paper, we determine the optimal distribution of parts among the existing stations using a class of adaptive search procedures called genetic algorithms (GAs). The class of GAs distinguish themselves from other optimization techniques by the use of concepts from population genetics to guide the search. It would be difficult to use classical approaches based on exact algorithms since the economic function here is rather complex and thus requires much computational effort. Moreover, as will be seen below, an economic function has no particular structure that we could take advantage of in assessing the effect of slightly modifying a solution. When the number of spare parts is small an exhaustive search of the optimal solution can be made. But the solution space increases very quickly as the number of spare parts increases and consequently heuristic methods must be used. We chose to use genetic type methods to solve the problem. It will be seen that they yield good results and can be useful in some practical problems.

This problem is related to the mathematical model METRIC (Multi-Echelon Technique for Recoverable Item Control). See for example Albright (1989), Demmy (1979), Demmy \& Presutti (1981), Graves (1985), Muckstadt (1973, 1978), Muckstadt \& Thomas (1980) and Sherbrooke (1967). METRIC was designed for military application at the weapon-system level where a particular item (part) may be demanded at several stations that are replenished by one central depot. The major difference with our model is the fact that in the METRIC model when an item becomes defective at a station, it can be replaced only by another item available at the station or at the central depot. Hence there is no lateral re-supply between stations which we consider in our model. Tedone (1989) describes the RAPS (Rotables Allocation and Planning System) system used by American Airlines and having similarities with the METRIC system. In this approach, the spare parts are distributed among stations according to weights based on the past history of failures at the stations. Other related papers are Cho \& Parlar (1991), Kim, Shin \& Park (2000) and Zorn, Deckro \& Lehmkuhl (1999). 
The problem formulation is introduced in Section 2 as well as the failure process of parts and the evaluation of the delay associated with a given solution. Like some classes of algorithms, GAs include several parameters and strategies leading to several variants. In Section 3, the genetic algorithm implementation and its variations are described. Section 4 includes the numerical experiments to identify efficient parameters for optimizing the distribution of parts. A conclusion follows in Section 5.

\section{Problem Formulation}

A different problem is formulated for each type of part. The mathematical formulation of the problem for a specific type of part is quite straightforward, but the evaluation of the objective function is complex and time consuming.

\subsection{Objective function and constraints}

Assume that an initial quantity of $N$ parts of a given type is available for redistribution among the stations and the maintenance shop for an airline. Denote

$A$ : the set of line stations indices $i$ (including the maintenance shop which is assumed to be located at one of the stations)

$P_{P}=\{i \in \mathrm{A} \backslash$ the airline is the provider of the part type at station $i\}$

$P_{A}=\{i \in \mathrm{A} \backslash$ the airline is a participant to the pool at station $i\}$

$M=\{i \in \mathrm{A} \backslash$ maintenance capacity exists for that part at station $i\}$

$\bar{M}=A-M$

Let $S$ be the total number of stations operated by the airline: $S=|A|$. Let $x_{i}$ be the number of parts allocated to stations $i \in A$ by the airline. The problem is to distribute the $N$ parts among the $S$ stations in order to minimize the expected cost of delay $D=D(X)$ due to unexpected failures, where $X=\left(x_{1}, x_{2}, \ldots, x_{s}\right)$. The problem then is to determine the optimal solution to the following optimization problem:

Minimize $D(X)$

$$
\text { s.t. }\left\{\begin{aligned}
\sum_{i=1}^{s} x_{i}=N & \\
x_{i} \geq 1 & \forall i \in P_{P} \\
x_{i}=0 & \forall i \in P_{A} \\
x_{i}=0 & \forall i \in \bar{M}
\end{aligned}\right.
$$

\subsection{Evaluation of the cost of delay $D(X)$}

In this section, we indicate how to determine the excepted cost of delay $D(X)$ given a specific allotment of spares $X$. The parts considered are all of the same type. 


\subsubsection{Average cost of delay per removal}

At station $i$, the total time delay $\delta_{i k}$ due to the failure of a part prior to flight departure $k$ includes the time to receive a serviceable part and the time to replace it on the aircraft. Since the replacement time of the part is constant regardless of the number of spares allocated at the station, it is not included in the time delay $\delta_{i k}$. Then if station $i$ has a spare part to replace the unserviceable one at the time of failure, the time delay is nil. Otherwise, the time delay $\delta_{i k}$ is the time it takes to receive a part through an incoming flight.

The flights considered in this study belong to a one week flight schedule. Consider the flight $k$, leaving from the origin station $i$. To determine the time delay $\delta_{i k}$ in the case where no spare part is available at the time of failure on flight $k$, we take into account each flight $i_{o} \rightarrow i$, leaving from origin station $i_{o}$, within 24 hours after the scheduled departure time of flight $k$, such that station $i_{o}$ can provide a spare part. Denote $\delta_{i k}\left(i_{o}\right)$ the time delay of flight $k$ if the spare part is provided at station $i$ by an incoming flight at station $i$ and originating from station $i_{o}$.

Hence $\delta_{i k}\left(i_{o}\right)$ is equal to the difference between the arrival time at $i$ of this flight leaving from $i_{o}$ and the schedule departure time of flight $k$.

To illustrate the evaluation process, consider the following time-space diagram where flights are illustrated with arcs:

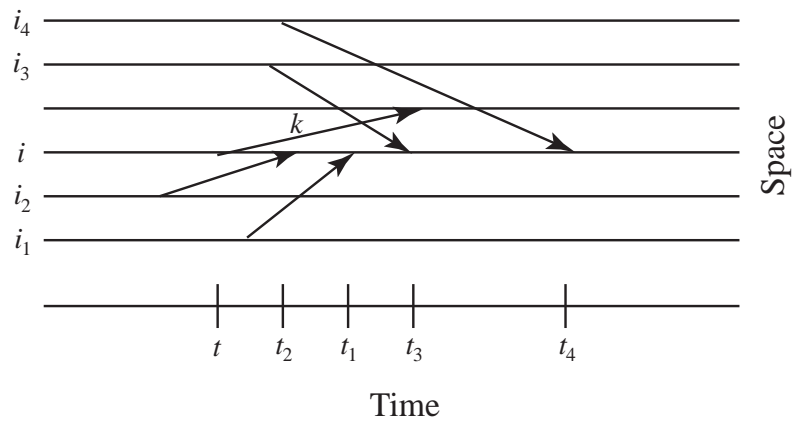

In this illustration, the flight leaving from $i_{2}$ cannot bring a spare part because its departure time is prior to the scheduled departure time of flight $k$. Furthermore, it follows that $\delta_{i k}\left(i_{j}\right)=\mathrm{t}_{\mathrm{j}}-\mathrm{t}$ for $\mathrm{j}=1,3,4$.

Recall that our model is a planning tool to be used at the tactical level. Hence we do not know precisely which station will have a spare part to provide station $i$ prior to the departure of flight $k$. Therefore we approximate $\delta_{i k}$ using the following underestimate:

$$
\delta_{i k}=\left\{\begin{array}{lll}
\min _{i_{o} \in \Gamma_{i k}}\left\{\delta_{i k}\left(i_{o}\right)\right\} & \text { if } & \Gamma_{i k} \neq 0 \\
1440 & \text { otherwise } &
\end{array}\right.
$$

where $\Gamma_{i k}=\left\{i_{o}: i_{o}\right.$ is the origin station of an incoming flight to $i$ such that its departure time is within 24 hours after the scheduled departure time of $k$, and $\left.x_{i_{o}}>0\right\}$. Here 1440 (24 hours $\times 60 \mathrm{~min}$ ) is the delay corresponding to waiting for the same flight the next day. 
The average cost of delay $d_{i}$ caused by the failure of a type of a part prior to any flight departure at station $i$ is evaluated as follows:

$$
d_{i}= \begin{cases}\frac{1}{|K|} \sum_{k \in K} \sqrt{\delta_{i k}} & \forall i \notin P_{A} \\ 0 & \forall i \in P_{A}\end{cases}
$$

where $K$ denotes the set of flights departing from station $i$ during the week, and that are operated with an aircraft using a type of part.

\subsubsection{Expected removals in a given period of time}

The expected number of removals for a type of parts is the expected number of times a part fails during that time period. Based on historical data, the mean time between removals (MTBR) $\tau$, is the average number of flying hours between two successive removals. A type of parts can belong to one or more types of aircraft. Let $Q(u)$ be the quantity of parts belonging to the aircraft of type $u$. Let $H(u)$ be the total hours operated by aircraft of type $u$ during the week. Then the total expected number of removals $\lambda$ for the part in a year is as follows:

$$
\lambda=\sum_{u} \frac{H(u) * Q(u) * 52}{\tau}
$$

In order to calculate the expected number of removals per station during a year, let $F(i, u)$ be the total number of flights per year with aircraft of type $u$, departing from station $i$. Then the total expected number of removals $\lambda_{\mathrm{i}}$ of that type of part at station $i$ during a year is

$$
\lambda_{i}=\frac{\lambda * \sum_{u} F(i, u) * Q(u)}{\sum_{i}\left(\sum_{u} F(i, u) * Q(u)\right)},
$$

and

$$
\lambda=\sum_{i} \lambda_{i}
$$

\subsubsection{Failure process}

When failures of parts occur at a rate less than 10 per year, removals are assumed to follow a Poisson process. Since $\lambda$ is the expected number of removals of the part during a year, the probability distribution of the number of removals $k_{\lambda \mathrm{t}}$ of the part in $[0, t]$ is as follows:

$$
P\left(k_{\lambda t} \leq U\right)=\sum_{r=0}^{U} P_{p}\left(k_{\lambda t}=r\right)=\sum_{r !}^{U} \frac{e^{\lambda t}(\lambda t)^{r}}{r !} .
$$

Similarly, the probability distribution of the number of removals of the part at station $i$ in $[0, t]$ is

$$
P\left(k_{\lambda_{i} t} \leq V\right)=\sum_{r=0}^{V} \frac{e^{-\lambda_{i} t}\left(\lambda_{i} t\right)^{r}}{r !}
$$


When failures of the part occur at a rate greater than or equal to 10 per year $(\lambda \geq 10)$, the number of removals in $[0, t]$ is assumed to have a normal distribution with mean equal to $\lambda t$ and standard deviation $\sigma=\sqrt{\lambda t}$ :

$$
P_{N}\left(k_{\lambda t, \sigma} \leq U\right)=P_{N}\left(Z \leq \frac{U-\lambda t}{\sigma}\right) .
$$

\subsubsection{Expected number of removals causing a delay}

The variable $x_{i}$ denotes the initial number of spares allocated at station $i$. However this number is reduced whenever a spare part is being used. Let $x_{i}(t)$ be the number of spare parts available at time of failure $t$ at station $i$ :

$$
x_{i}(t) \leq x_{i} \quad \forall t
$$

Two cases have to be considered to calculate the expected delay for station $i$ according to the fact that $x_{i}=0$ or $x_{i}>0$.

If no spare part is allocated at station $i$ then $x_{i}=x_{i}(t)=0, \forall \mathrm{t}$. In this case, the expected number of removals at station $i$ causing a delay is equal to the expected number of removals $\lambda_{i}$ (see subsection 2.2.2) at station $i$.

If $x_{i}>0$, let $\mu_{i}$ be the expected number of removals at station $i$ per year causing a delay. Note that $x_{i} \leq \lambda_{i}, \forall i$. Let $\mu_{i}(t)$ be the expected number of removals at station $i$ during a time period $t$, causing a delay. Then given the allotment $\mathrm{x}_{i}$,

$$
\begin{aligned}
\mu_{i}(t)= & 0 * P\left(k_{\lambda_{i} t} \leq x_{i}\right)+1 * P\left(k_{\lambda_{i} t}=x_{i}+1\right)+2 * P\left(k_{\lambda_{i} t}=x_{i}+2\right) \ldots \\
= & \sum_{s=x_{i}}^{\infty}\left(s-x_{i}\right) P\left(k_{\lambda_{i} t}-s\right) \\
= & \sum_{s=x_{i}}^{\infty} s P\left(k_{\lambda_{i} t}=s\right)-x_{i} \sum_{s=x_{i}}^{\infty} P\left(k_{\lambda_{i} t}=s\right) \\
= & \left(\lambda_{i} t-\sum_{s=0}^{x_{i}-1} s P\left(k_{\lambda_{i} t}=s\right)\right)-x_{i}\left(1-\sum_{s=0}^{x_{i}-1} P\left(k_{\lambda_{i} t}=s\right)\right) \\
= & \sum_{s=0}^{x_{i}-1}\left(x_{i}-s\right) P\left(k_{\lambda_{i} t}=s\right)-\left(x_{i}-\lambda_{i} t\right)
\end{aligned}
$$

To evaluate $\mu_{i}$ we suppose that the stock runs out at station $i$ during replenishment time $R$ required to receive a serviceable part at the station from the shop in replacement of its unserviceable one. Now, $R$ is equal to the transit time $T_{T}$ when the shop has spare part in stock. Otherwise, $R=T_{T}+T_{A}$ where $\mathrm{T}_{A}$ (Turn Around Time) is the time required to repair a defective part at the maintenance shop.

If we denote

$$
\begin{aligned}
& P\left(R=T_{T}\right)=L \\
& P\left(R=T_{T}+T_{A}\right)=1-L
\end{aligned}
$$


it follows that the expected number of removals $\mu_{i}$ of the part causing a delay at station $i$ is as follows:

$$
\mu_{i}=L * \mu_{i}\left[T_{T}\right]+(1-L) \mu_{i}\left[T_{T}+T_{A}\right]
$$

In order to simplify the computations, the expected number of removals at the shop (located at station $M)$ is taken to be equal to the total expected number of removals $\lambda\left(\lambda=\sum_{i} \lambda_{i}\right)$.

The probability $L$ is computed using the Poisson distribution function when $\lambda<10$ or using the Normal distribution when $\lambda \geq 10$. Given the initial quantity of spare parts $x_{D}$ and the quantity of spare parts at the time of failure $x_{M}(t)$ at the shop, the probability $L$ is given by

$$
L=P\left(x_{M}(t)>0\right)=P\left(R=T_{T}\right)
$$

and it is approximated by

$$
P\left(k_{\lambda T} \leq x_{D}\right)
$$

that is the probability that the expected number of removals from the maintenance shop during the time required to repair a part is less than or equal to the number of spare parts at station $\mathrm{M}$.

\subsection{Average cost of delay}

Let

$$
J_{i}=\left\{\begin{array}{lll}
0 & \text { if } & x_{i}>0 \\
1 & \text { if } & x_{i}=0
\end{array}\right.
$$

Then $D(X)$, the expected total cost of delay caused by a failure given the allotment $X=\left(x_{1}, x_{2}, \ldots, x_{s}\right)$, is as follows:

$$
D(X)=\sum_{i=1}^{s} d_{i}\left(J_{i} \lambda_{i}+\left(1-J_{i}\right) \mu_{i}\right) .
$$

It is worthy of note that we have to scan the weekly schedule file in order to evaluate the function $D(X)$ for each solution $X$. Since the schedule may include a large number of flights, it follows that the evaluation of function $D(X)$ is in general costly and computationintensive to run.

\section{Solution Approach Using Genetic Algorithms}

Conventional computational techniques, or exact algorithms, are difficult to apply to our optimization problem since, as mentioned before, the objective function is very complex and time consuming to evaluate. Furthermore, it does not include any nice structure to take advantage of in order to easily evaluate the effect on its value induced by a slight modification of the solution. If the search space is not too large, one can usually develop an enumeration search strategy with appropriate heuristic cutoffs, thus keeping the computation time under control. Otherwise if the search space is large, exhaustive search techniques are computationally too 
expensive. Indeed, for a part with an allotment of $N$ spare parts to be allocated at $S$ feasible stations, the total number of possible solutions $F(S, N)$ is as follows:

$$
F(S, N)=\left(\begin{array}{c}
N+S-1 \\
N
\end{array}\right)=\frac{(N+S-1) !}{N !(S-1) !} .
$$

Hence if $S=15$ and $N=6$, then $F(S, N)=38,760$, and if $N$ increases to 11 , the search space can be as large as 4,457,400 solutions.

Heuristic or approximate algorithms are often preferred in this case to quickly generate an approximate optimal solution. Genetic algorithms are population based heuristic techniques relying on the genetic inheritance, and allowing a more exhaustive search of the solution space. Some references on Genetic algorithms are the following: Buckles \& Petry (1992), Davis (1987, 1991), Fox \& Landi (1970), Goldberg (1989), Holland (1975) and Michalewicz (1992).

\subsection{Presentation of the Algorithm}

An initial population of $n$ chromosomes or solutions is created. All $n$ chromosomes are evaluated. At each generation, $n$ chromosomes are chosen for reproduction from the current population. The selection is completed according to the proportional selection inducing a random process ensuring that the expected number of times a chromosome is chosen is approximately proportional to that chromosome's performance relative to the rest of the population. Hence the same chromosome can be selected more than once. New chromosomes are generated by means of genetic recombination operators. The recombination operator is called crossover, and in general, it generates two new candidate solutions called offsprings. Then some perturbations are applied to the offsprings with low frequency via the mutation operator. All the new offsprings are added to the set of parents to create a temporary population of size $2 n$. $n$ chromosomes are selected from the temporary population via proportional selection, to create a new generation of size $n$. This process if repeated until a maximum number of generations is reached. The genetic algorithm used in our study is summarized in Figure 1:

1. Create a temporary initial population of $n$ chromosomes (Generation 0 )

2. Evaluate the fitness of each chromosome

Repeat while the number of generations is less than the maximum number of generations:

3. Select $n$ parents from the temporary population via proportional selection to create a new generation

Repeat until all parents are selected ( $n$ offsprings are created):

4. Choose a pair of parents for mating. Apply the crossover to create two offsprings

5. Process each offspring by the mutation operator

End repeat

6. Evaluate the fitness of each offspring

7. Add the offsprings to the set of parents to form a new temporary population of size $2 n$

End repeat

Figure 1 - Basic lines of the proposed GA 


\subsection{Solution encoding}

A crucial operation in using a genetic algorithm to solve a problem is the way of representing or encoding a solution. The technique for encoding a solution may vary from one problem to another. In earlier works, researchers used to encode all solutions as bit strings, regardless of the problem. Later, other types of encoding techniques were used. In our case, a bit string encoding is not natural. Indeed, a solution vector $X=\left(x_{1}, x_{2}, \ldots, x_{s}\right)$ (the phenotype form of the solution) is encoded into a genotype form of dimension $N$, the initial number of spare parts. Each vector component contains the station index where one part is allocated. If more than one part is allocated at the station, then the station index is repeated for the next vector component. Denote $G=\left(g_{1}, g_{2}, \ldots, g_{N}\right)$ the genotype representation of a vector solution, where $g_{1} \in A, \quad 1=1, \ldots, N$, and $A$ is a set of all stations. For example, for a part where $N=6$ and $S=60$, let $X=\left(x_{1}, x_{2}, x_{3}, \ldots, x_{s}\right)=(1,0,2,0, \ldots, 0,3)$, the genotype vector $G$ is represented as $\mathrm{G}=(1,3,3,60,60,60)$.

The fitness of a chromosome solution $G$ denoted as $f(G)$, is defined to be the inverse of the cost of delay function $D(X)$ :

$$
f(G)=\frac{1}{D(X)}
$$

\subsection{Population}

The population size $n$ is the number of solutions or chromosomes per population. The population size remains constant from generation to generation. Population size is a fundamental parameter of any GA. If the selected population size is too small, the algorithm may converge too quickly but not necessarily to a global or local optimal solution. On the other hand, a population with too many members offers a larger pool of diverse solutions, but might result in long waiting times for significant improvement.

The key feature of GAs is their ability to take advantage of the cumulated information about an initially unknown search space in order to bias subsequent search into useful subspaces. Most genetic algorithm implementations do begin with random populations. However, if problem-specific knowledge is available to indicate interesting regions of the feasible domain, then it should be used to guide the process. Initial solutions generated according to problem specific-knowledge are called seeded solutions. Only the initial population is seeded with good initial members.

For the Aircraft Spare Parts problem, preliminary results indicate that the time delay is smaller when more parts are assigned to stations with high frequency of departures. Indeed, consider a problem where $N=8$ and $S=10$. Table 1 and Figure 2 illustrate the delay for ten different solutions where the total number of parts is assigned to one station, and none to the other 9 stations. In general, the delay decreases as the departure frequency of the station where all the spare parts are allocated increases. 
Table 1 - First situation: Seeded Solutions

\begin{tabular}{|c|c|c|c|c|}
\hline Solution & Station & Frequency & Parts @ station & Delay (min.) \\
\hline$\# 1$ & YYZ & 317 & 8 & 97 \\
$\# 2$ & YUL & 134 & 8 & 149 \\
$\# 3$ & YVR & 103 & 8 & 187 \\
$\# 4$ & YYC & 101 & 8 & 178 \\
$\# 5$ & YHZ & 79 & 8 & 180 \\
$\# 6$ & YWG & 79 & 8 & 196 \\
$\# 7$ & YOW & 53 & 8 & 224 \\
$\# 8$ & YEG & 7 & 8 & 261 \\
$\# 9$ & SFO & 7 & 8 & 246 \\
$\# 10$ & MIA & 1 & 8 & 257 \\
\hline
\end{tabular}

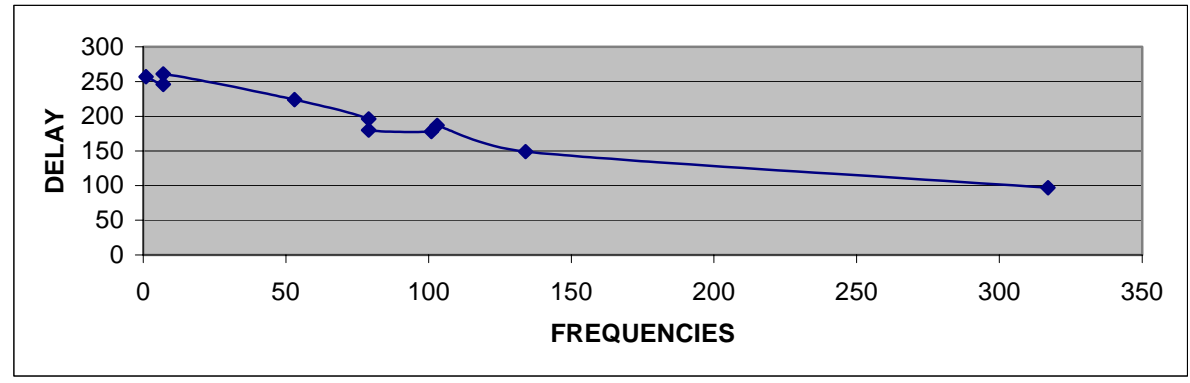

Figure 2 - First situation: Frequency-Delay Relationship

Since the time delay at a station does not depend uniquely on the number of spare parts but also on the providing from the neighboring stations, another situation is analyzed. Here, we use the same part with the same feasible stations, but we assume that 72 spare parts are available. Ten different solutions are evaluated where no spare part is assigned to one station and 8 spares to each of the other 9 stations. The results in Table 2 and Figure 3 indicate that, in general, the time delay increases with the augmenting of the departure frequency of the station having no spare part.

Table 2 - Second situation: Seeded Solutions

\begin{tabular}{|c|c|c|c|c|}
\hline Solution & Station & Frequency & Parts @ station & Delay (min.) \\
\hline$\# 1$ & YYZ & 317 & 0 & 64 \\
$\# 2$ & YUL & 134 & 0 & 40 \\
$\# 3$ & YVR & 103 & 0 & 43 \\
$\# 4$ & YYC & 101 & 0 & 43 \\
$\# 5$ & YHZ & 79 & 0 & 38 \\
$\# 6$ & YWG & 79 & 0 & 40 \\
$\# 7$ & YOW & 53 & 0 & 40 \\
$\# 8$ & YEG & 7 & 0 & 35 \\
$\# 9$ & SFO & 7 & 0 & 35 \\
$\# 10$ & MIA & 1 & 0 & 34 \\
\hline
\end{tabular}




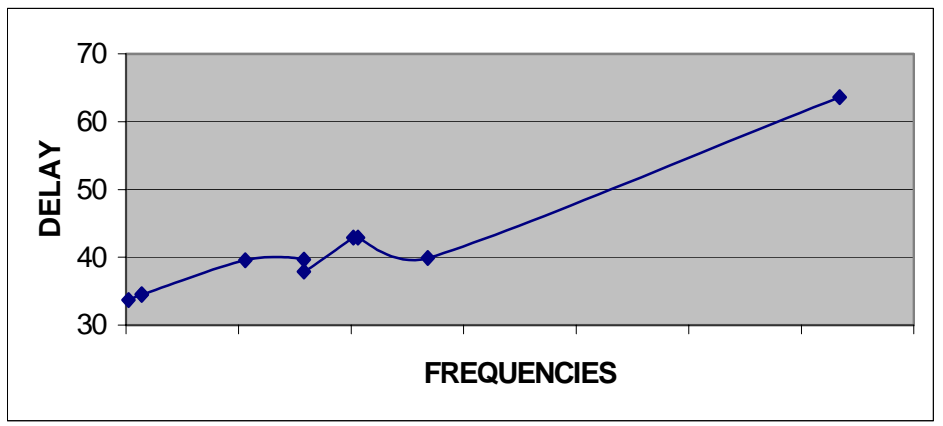

Figure 3 - Second situation: Frequency-Delay Relationship

Relying on these observations, the initial population can be partially seeded using frequency of departures per station as a parameter. In this case, some seeded chromosomes are included in the initial population. Each seeded chromosome is specified according to the following proportional selection process. First, the stations are ordered in some sequence. To determine the station in each position of the chromosome, a random positive integer smaller than or equal to the total number of flights is generated. Then we select the first station in the sequence such that the sum of its frequency and those of the previous stations in the sequence is greater than or equal to the random number. This proportional selection process ensures that the expected number of times a station is chosen is approximately proportional to that station frequency relative to the rest of the stations. Station indices per generated solution are sorted in ascending order, i.e. for an initial solution $G=\left(g_{1}, g_{2}, \ldots, g_{N}\right), g_{l} \leq g_{m}$ $\forall l \leq m$. In Section 4, numerical results are used to analyze the influence of the percentage of seeded solutions in the initial population and the effect of sorted initial solutions.

At each generation, $n$ new parents are selected from the temporary population. Each new parent is selected according to a proportional selection process, also known as the roulette wheel parent selection in order to ensure that the expected number of times a chromosome is chosen is approximately proportional to its performance relative to the rest of the population. First the fitness values of all chromosomes in the current population is determined and the chromosomes are ordered in some sequence. Then the following procedure is applied to determine each of the $n$ parents:

1. Generate a random number between 0 and the total of the fitness values.

2. Select the first chromosome in the sequence whose fitness value added to the sum of the fitness values of the previous chromosomes in the sequence is greater than or equal to the random number generated at step 1.

As mentioned before, proportional selection process is applied to the temporary population formed by $n$ parents and their $n$ offsprings in order to form a new generation of $n$ chromosomes (with the exception of the temporary initial solution which contains only $n$ initial solutions). The advantage of this selection technique is that it directly promotes reproduction of the fittest population members. Several other replacement strategies are currently used where only a portion of the population is kept from one generation to another. In Section 4, numerical results are used to evaluate the efficiency of other strategies, by keeping a small percentage of best performing chromosomes from generation to generation and selecting the rest of the population via proportional selection. 


\subsection{Operators}

Crossover is an extremely important component of genetic algorithm. It is regarded as the distinguishing feature of GAs and as a critical accelerator of the search process. Crossover is a structured yet randomized information exchange between two chromosomes. The role of the crossover operator is to combine two solutions into an offspring that shares characteristics from both parents. The more widely used operators are the 1-point, the 2-point, and the uniform crossovers. Since in our problem the dimension of the vector solution varies between 3 and 11, only the 1-point and the uniform operators are compared.

In the 1-point crossover, we randomly select a cut point in the parents. Then offsprings are generated by combining the genetic material of one parent on the left-hand side of the cut point with that of the other parent for the positions on the right-hand side (and including the cut point). Figure 4 illustrates an example where two parents are combined via the 1-point crossover.

\begin{tabular}{lccccccccc}
\hline \hline Parent 1 & MIA & SFO & YEG & YHZ & YVR & YWG & YUL & YUL & YYZ \\
Parent 2 & SFO & YEG & YHZ & YWG & YWG & YUL & YYZ & YYZ & YYZ \\
\hline Random & & & & & & $\uparrow$ & & & \\
Position & & & & & & & & & \\
\hline Offspring 1 & MIA & SFO & YEG & YHZ & YVR & YUL & YYZ & YYZ & YYZ \\
Offspring 2 & SFO & YEG & YHZ & YWG & YWG & YWG & YUL & YUL & YYZ \\
\hline
\end{tabular}

Figure 4 - 1-point Crossover

In the uniform crossover operator, a random bit string of size $N$ is generated. The station in each position of the offsprings is specified according to the bit string as follows: if there is a 0 in a given position of the bit string, the offspring 1 inherits the station of parent 2 for the same position, and offspring 2, that of parent 1 . If there is a 1 instead, offspring 1 inherits the station of parent 1 , and offspring 2, that of parent 2. Figure 5 illustrates the combination of two parents using a uniform crossover.

Mutation occurs after crossover is applied, but only a small percentage of the time. Indeed, with a $1 \%$ probability, every component of each vector solution is replaced by a random feasible station.

\begin{tabular}{lccccccccc}
\hline \hline Parent 1 & MIA & SFO & YEG & YHZ & YVR & YWG & YUL & YUL & YYZ \\
Parent 2 & SFO & YEG & YHZ & YWG & YWG & YUL & YYZ & YYZ & YYZ \\
\hline $\begin{array}{l}\text { Random Bit } \\
\text { String }\end{array}$ & 0 & 1 & 1 & 1 & 0 & 1 & 0 & 0 & 0 \\
\hline $\begin{array}{l}\text { Offspring 1 } \\
\text { Offspring 2 }\end{array}$ & SFO & SFO & YEG & YHZ & YWG & YWG & YYZ & YYZ & YYZ \\
\hline
\end{tabular}

Figure 5 - Uniform Crossover 


\section{Numerical Results}

Choosing a suitable population size, the right combination operator or even a reasonable stopping criteria are key decisions faced by all genetic algorithm users. Parameter settings greatly influence performance. Some of the parameters are tested individually and some dependent parameters are tested when combined. In order to test one parameter, all the others are fixed and the tested parameter is assigned various values.

Three different problems with 3, 6 and 9 spare parts available are used to analyze the parameter values. Each part type belongs to a different aircraft type, but each fleet covers a similar network of stations. The number of parts per aircraft, the number of flight departures per week, and the number of feasible stations covered by the fleet type are summarized in Table 3.

Table 3 - Part types

\begin{tabular}{|c|c|c|c|c|}
\hline $\begin{array}{c}\text { Problem } \\
\text { number }\end{array}$ & $\begin{array}{c}\text { Initial } \\
\text { Quantity } N\end{array}$ & $\begin{array}{c}\text { Quantity per } \\
\text { aircraft }\end{array}$ & $\begin{array}{c}\text { Departures } \\
\text { per week }\end{array}$ & $\begin{array}{c}\text { Feasible } \\
\text { stations }\end{array}$ \\
\hline 1 & 3 & 2 & 864 & 10 \\
2 & 6 & 9 & 410 & 10 \\
3 & 9 & 2 & 1222 & 12 \\
\hline
\end{tabular}

For each test, each problem is solved 10 times. In the different tables summarizing the results, the "Best Value" column indicates the minimum cost of delay achieved among the total number of runs. The average cost of delay for the 10 tests is also indicated together with the confidence interval having a $95 \%$ confidence level. A confidence interval noted $\pm \beta$ corresponds to $A(X) \pm \beta$, where $A(X)$ is the average of the 100 tests. The tables also include the number of times (out of the 10 runs) that the best value is reached, the average number of solutions generated per test, and the average time of execution. Note that the computations were executed on a PC based Toshiba 4030 CDS, $233 \mathrm{MHz}$.

\subsection{Stopping criteria}

The stopping criterion used in our tests consists of stopping the process when the number of iterations reaches $10 *\lfloor N / 2\rfloor$, a number proportional to the number $N$ of spare parts available for the problem. This stopping criterion results in exploring a reasonable number of solutions and obviously not exceeding the total number of feasible solutions.

\subsection{Population size}

The population size $n$ remains constant from generation to generation, and it is directly proportional to the length of the genotype vector $N$ (the number of spare parts available): $n=\alpha N$, where $\alpha$ is a positive integer. Three different values of $\alpha$ are tested, and the results are summarized in Table 4 . The parameters are fixed as follows:

Population size test: $n=3 N$ or $n=5 N$ or $n=7 N(\alpha=3,5$ or 7$)$.

Mutation operator rate: $1 \%$

Percentage of seeded solutions in initial population: $50 \%$ 
Percentage of best solutions for new generation: $10 \%$

Crossover operator: Uniform

Initial solutions are sorted: $G=\left(g_{1}, g_{2}, \ldots, g_{\mathrm{N}}\right), g_{1} \leq g_{m} \quad \forall l \leq m$.

As far as solution quality is concerned, the results are quite similar when $\alpha$ is equal to 3 and 5. However, the larger the population size, the more solutions are evaluated, and therefore solution time increases. Hence, the population size $n=5 N$ is selected.

Table 4 - Population Size Test Results

\begin{tabular}{ccccccrr}
\hline $\begin{array}{c}\text { Problem } \\
\text { Size } N\end{array}$ & $\begin{array}{c}\text { Best } \\
\text { value }\end{array}$ & $\begin{array}{c}\text { Population } \\
\text { size } n\end{array}$ & $\begin{array}{c}\text { Avg. cost } \\
\text { of delay } \\
A(X)\end{array}$ & $\begin{array}{c}\text { Confidence } \\
\text { interval }\end{array}$ & $\begin{array}{c}\text { Total best } \\
\text { solutions }\end{array}$ & $\begin{array}{c}\text { Solutions } \\
\text { generated }\end{array}$ & $\begin{array}{c}\text { Times } \\
(\mathrm{mns})\end{array}$ \\
\hline 3 & \multirow{2}{*}{17.80} & $3 N$ & 18.33 & \pm 0.59 & 6 & 90 & 2.2 \\
& & $5 N$ & 17.82 & \pm 0.02 & 8 & 150 & 3.6 \\
& & $7 N$ & 18.02 & \pm 0.27 & 8 & 210 & 4.9 \\
\hline 6 & \multirow{2}{*}{7.20} & $3 N$ & 7.29 & \pm 0.06 & 5 & 539 & 7.2 \\
& & $5 N$ & 7.24 & \pm 0.04 & 7 & 900 & 11.8 \\
& & $7 N$ & 7.21 & \pm 0.02 & 9 & 1260 & 16.3 \\
\hline 9 & 91.00 & $3 N$ & 92.41 & \pm 1.48 & 5 & 1079 & 19.0 \\
& & $5 N$ & 91.27 & \pm 0.50 & 9 & 1800 & 30.4 \\
& & $7 N$ & 91.00 & \pm 0.00 & 10 & 2520 & 41.8 \\
\hline
\end{tabular}

\subsection{Seeded initial population}

As it has been indicated in Section 3, the delay is inversely proportional to the departure frequency at the station when all spare parts are allocated to the same station. However, the presence of too many highly fit chromosomes in the initial population may result in premature termination at a local optimum. In order to identify the best strategy, three values are tested for the percentages of seeded initial solutions:

Percentage of seeded solutions in initial population: $20 \%$ or $50 \%$ or $80 \%$

Population size $n=5 N$

Mutation operator rate: $1 \%$

Percentage of best solutions for new generation: $10 \%$

Crossover operator: Uniform

Initial solutions are sorted: $G=\left(g_{1}, g_{2}, \ldots, g_{N}\right), g_{l} \leq g_{m} \quad \forall l \leq m$.

The results in Table 5 indicate that in general, an initial population with $50 \%$ seeded solutions seems to be a good choice. Figure 6 illustrates how the cost of delay decreases during the resolution for each percentage $(20 \%, 50 \%, 80 \%)$ for problem 1 with $N=3$. Each point on the graph represents the average cost of delay for the $n$ solutions. As expected, for the initial population with $20 \%$ seeded solutions (i.e. $80 \%$ random solutions), the average cost is initially higher, and it takes longer to converge to a minimum. However, for the initial population with $80 \%$ seeded solutions, the average cost is initially better and it converges rapidly, reaching sometimes a local minimum. With a $50 \%$ seeded initial population, the algorithm seems to converge to optimal solutions more likely than with $20 \%$ or $80 \%$. 
Table 5 - Initial Population Test Results

\begin{tabular}{cccccccc}
\hline $\begin{array}{c}\text { Problem } \\
\text { Size } N\end{array}$ & $\begin{array}{c}\text { Best } \\
\text { value }\end{array}$ & $\begin{array}{c}\text { Seed } \\
\text { percentage }\end{array}$ & $\begin{array}{c}\text { Avg. cost } \\
\text { of delay } \\
A(X)\end{array}$ & $\begin{array}{c}\text { Confidence } \\
\text { interval }\end{array}$ & $\begin{array}{c}\text { Total best } \\
\text { solutions }\end{array}$ & $\begin{array}{c}\text { Solutions } \\
\text { generated }\end{array}$ & $\begin{array}{c}\text { Times } \\
(\mathrm{mns})\end{array}$ \\
\hline 3 & \multirow{2}{*}{17.80} & $20 \%$ & 18.29 & \pm 0.50 & 5 & 176 & 5.2 \\
& & $50 \%$ & 17.80 & \pm 0.00 & 10 & 150 & 4.3 \\
& & $80 \%$ & 18.23 & \pm 0.41 & 7 & 160 & 4.7 \\
\hline 6 & \multirow{2}{*}{7.20} & $20 \%$ & 7.25 & \pm 0.05 & 7 & 930 & 12.6 \\
& & $50 \%$ & 7.24 & \pm 0.04 & 7 & 930 & 12.1 \\
& & $80 \%$ & 7.26 & \pm 0.06 & 7 & 900 & 11.7 \\
\hline 9 & 91.00 & $20 \%$ & 91.47 & \pm 0.48 & 7 & 1804 & 30.0 \\
& & $50 \%$ & 91.27 & \pm 0.50 & 9 & 1804 & 30.5 \\
& & $80 \%$ & 91.24 & \pm 0.30 & 8 & 1804 & 30.1 \\
\hline
\end{tabular}

\subsection{Parent selection}

At each iteration, a small percentage of best performing chromosomes selected as parents at the preceding iteration is kept, and the others are selected according to proportional selection. The results for four different percentages are summarized in Table 6.

Percentage of best solutions: $0 \%$ or $4 \%$ or $10 \%$ or $25 \%$

Population size $n=5 \mathrm{~N}$

Mutation operator rate: $1 \%$

Percentage of seeded solutions in initial population: $50 \%$

Crossover operator: Uniform

Initial solutions are sorted: $\mathrm{G}=\left(g_{1}, g_{2}, \ldots, g_{N}\right), g_{l} \leq g_{m} \quad \forall l \leq m$.

When the best performing solutions are not forced to remain from generation to generation (best solutions percentage $=0 \%$ ), they tend to be lost. Hence, the results in Table 6 indicate that the algorithm takes longer to regenerate good performing solutions. However, when the percentage selection is $10 \%$ or $25 \%$, the results for all 3 problem-tests are very good. For the problem when $N=3$, a percentage selection of $25 \%$ seems to be a bit too aggressive as 3 out of 10 tests converge to a local minimum.

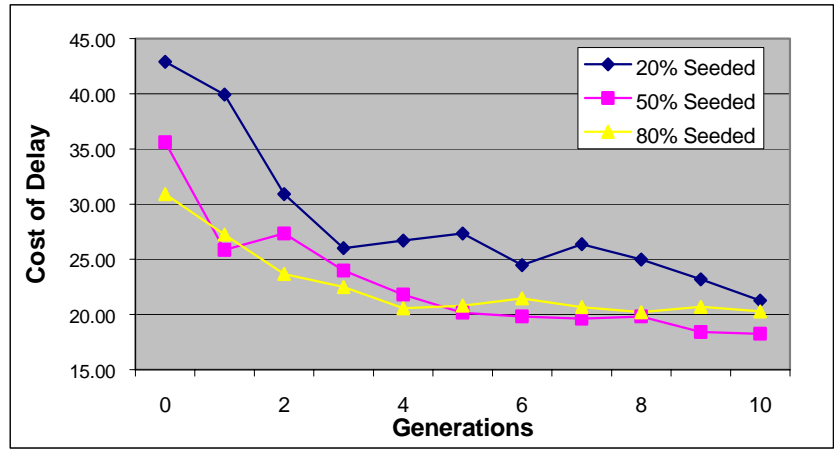

Figure 6 - Seeded Population Comparison 


\subsection{Crossover operator}

The uniform crossover and the 1-point crossover operators are compared. An additional component is added to the tests of the crossover operators in order to analyze the relevance of sorting the chromosomes in the initial solutions. Both parameters are tested jointly since the value of one can be dependent on the other. The results summarized in Table 7 are obtained using the following parameters:

Crossover operators: Uniform or 1-point.

Initial solutions are sorted: $\mathrm{G}=\left(g_{1}, g_{2}, \ldots, g_{N}\right), g_{l} \leq g_{m} \quad \forall l \leq m$ or non-sorted.

Percentage of best solutions: $10 \%$

Population size $n=5 \mathrm{~N}$

Mutation operator rate: $1 \%$

Percentage of seeded solutions in initial population: $50 \%$

The results for problem 2 where $N=6$ are not very conclusive, since the average values for the 4 combinations of the two parameters are very similar and the confidence intervals are short. Now, for the problems with $N=3$ and $N=9$, the results in Table 7, indicate that sorting the initial solutions (i.e. sorting the station indices within each chromosome) seems to allow generating better chromosomes and therefore the algorithm converges to better solutions.

Table 6 - Offsprings Selection Test Results

\begin{tabular}{|c|c|c|c|c|c|c|c|}
\hline $\begin{array}{c}\text { Problem } \\
\text { Size } N\end{array}$ & $\begin{array}{c}\text { Best } \\
\text { value }\end{array}$ & $\begin{array}{c}\% \text { Best } \\
\text { solutions }\end{array}$ & $\begin{array}{l}\text { Avg. cost } \\
\text { of delay } \\
\quad A(X)\end{array}$ & $\begin{array}{c}\text { Confidence } \\
\text { interval }\end{array}$ & $\begin{array}{l}\text { Total best } \\
\text { solutions }\end{array}$ & $\begin{array}{l}\text { Solutions } \\
\text { generated }\end{array}$ & $\begin{array}{l}\text { Times } \\
(\mathrm{mns})\end{array}$ \\
\hline \multirow[t]{4}{*}{3} & 17.80 & $0 \%$ & 18.23 & \pm 0.30 & 5 & 176 & 4.00 \\
\hline & & $4 \%$ & 17.91 & \pm 0.18 & 8 & 176 & 4.60 \\
\hline & & $10 \%$ & 17.80 & \pm 0.00 & 10 & 176 & 4.25 \\
\hline & & $25 \%$ & 18.01 & \pm 0.40 & 7 & 176 & 4.30 \\
\hline \multirow[t]{4}{*}{6} & 7.20 & $0 \%$ & 7.59 & \pm 0.13 & 2 & 930 & 12.70 \\
\hline & & $4 \%$ & 7.28 & \pm 0.05 & 4 & 930 & 12.20 \\
\hline & & $10 \%$ & 7.24 & \pm 0.04 & 7 & 930 & 12.13 \\
\hline & & $25 \%$ & 7.22 & \pm 0.02 & 8 & 918 & 12.00 \\
\hline \multirow[t]{4}{*}{9} & 91.00 & $0 \%$ & 94.71 & \pm 2.66 & 4 & 1804 & 30.02 \\
\hline & & $4 \%$ & 92.17 & \pm 0.99 & 6 & 1804 & 30.00 \\
\hline & & $10 \%$ & 91.27 & \pm 0.50 & 9 & 1804 & 30.50 \\
\hline & & $25 \%$ & 91.00 & \pm 0.00 & 10 & 1804 & 31.50 \\
\hline
\end{tabular}


Table 7 - Crossover Test Results

\begin{tabular}{|c|c|c|c|c|c|c|c|}
\hline $\begin{array}{c}\text { Problem } \\
\text { Size } N\end{array}$ & $\begin{array}{c}\text { Best } \\
\text { value }\end{array}$ & $\begin{array}{c}\text { Crossover } \\
\text { operator }\end{array}$ & $\begin{array}{c}\text { Sorted } \\
\text { initial } \\
\text { solutions }\end{array}$ & $\begin{array}{c}\text { Avg. Cost } \\
\text { of delay } \\
A(X)\end{array}$ & $\begin{array}{l}\text { Confidence } \\
\text { interval }\end{array}$ & $\begin{array}{c}\text { Total } \\
\text { best } \\
\text { solution }\end{array}$ & $\begin{array}{l}\text { Times } \\
(\mathrm{mns})\end{array}$ \\
\hline \multirow[t]{4}{*}{3} & \multirow[t]{4}{*}{17.80} & Uniform & No & 18.06 & \pm 0.26 & 4 & 4.50 \\
\hline & & 1-Point & No & 17.92 & \pm 0.33 & 8 & 4.10 \\
\hline & & Uniform & Yes & 17.80 & \pm 0.00 & 10 & 4.25 \\
\hline & & 1-Point & Yes & 17.93 & \pm 0.33 & 7 & 4.60 \\
\hline \multirow[t]{4}{*}{6} & \multirow[t]{4}{*}{7.20} & Uniform & No & 7.25 & \pm 0.10 & 8 & 11.60 \\
\hline & & 1-Point & No & 7.22 & \pm 0.04 & 8 & 11.60 \\
\hline & & Uniform & Yes & 7.24 & \pm 0.07 & 7 & 12.13 \\
\hline & & 1-Point & Yes & 7.27 & \pm 0.10 & 6 & 11.60 \\
\hline \multirow[t]{4}{*}{9} & \multirow[t]{4}{*}{91.00} & Uniform & No & 94.71 & \pm 0.77 & 7 & 30.30 \\
\hline & & 1-Point & No & 92.02 & \pm 1.28 & 5 & 30.90 \\
\hline & & Uniform & Yes & 91.27 & \pm 0.50 & 9 & 30.50 \\
\hline & & 1-Point & Yes & 91.36 & \pm 0.55 & 7 & 31.50 \\
\hline
\end{tabular}

Table 8 - Test Results for Various Part Types

\begin{tabular}{ccccc}
\hline $\begin{array}{c}\text { Problem } \\
\text { Size } N\end{array}$ & $\begin{array}{c}\text { Best } \\
\text { value }\end{array}$ & $\begin{array}{c}\text { Avg. Cost } \\
\text { of delay } \\
A(X)\end{array}$ & $\begin{array}{c}\text { \% Variance } \\
\text { from best } \\
\text { value }\end{array}$ & $\begin{array}{c}\text { Total best } \\
\text { solutions out } \\
\text { of 10 tests }\end{array}$ \\
\hline 3 & 17.80 & 17.80 & $0.0 \%$ & 10 \\
4 & 11.80 & 11.93 & $1.1 \%$ & 9 \\
5 & 26.20 & 26.28 & $0.3 \%$ & 6 \\
6 & 7.20 & 7.24 & $0.6 \%$ & 7 \\
7 & 88.80 & 88.99 & $0.2 \%$ & 9 \\
8 & 34.70 & 35.15 & $1.3 \%$ & 7 \\
9 & 91.00 & 91.27 & $0.3 \%$ & 9 \\
10 & 59.10 & 59.10 & $0.0 \%$ & 10 \\
11 & 62.40 & 62.48 & $0.1 \%$ & 7 \\
\hline
\end{tabular}

The uniform crossover is more efficient than the 1-point crossover in 5 out of 6 cases, as shown in the "Total Best Solutions" column. This result is consistent with the one obtained in Syswerda (1989) (Section 4.6).

\subsection{Numerical results for 9 problems}

Other results are obtained for other problems including different numbers of spare parts $(3 \leq N \leq 11)$, and belonging to different aircraft types. Table 8 includes the average results taken over 10 tests for each problem when the parameter values are fixed according to the preceding analysis. These results are very encouraging, showing the good performance of the Genetic Algorithms to solve the Spares problem. 


\section{Conclusion}

Since the problem includes a small number of constraints, Genetic Algorithms seem to be very appropriate to deal with the Aircraft Spare Parts problem. The percentage variance of the average cost from the best value varies between $0.0 \%$ to $1.3 \%$. This is the case for all problem sizes, for various part types and aircraft types.

It is worth noting that the algorithm is computation intensive to run. The evaluation of a solution is making the algorithm computationally expensive, as the delay is estimated for every single flight of the week. Should this optimization be required on a frequent basis, or within few hours, the cost of delay could be simulated instead of being fully evaluated. By doing so, the execution would be reduced.

Of course comparisons should be made with other approaches. But this would be the object of another paper.

\section{Acknowledgements}

This research was supported by NSERC grants OGP0003736 and OGP0008312.

The authors thank the referees for their helpful comments.

\section{References}

(1) Albright, S.C. (1989). An approximation to the Stationary Distribution of a Multiechelon Repairable Item Inventory System with Finite Sources and Repair Channels. Nav. Res. Logist. Quart., 36, 179-195.

(2) Buckles, B. \& Petry, F. (1992). Genetic Algorithms. IEEE Computer Society Press, Los Alamitos, California.

(3) Cho, D.I. \& Parlar, M. (1991). A Survey of Maintenance Models for Multi-Unit Systems. Euro. Jour. Oper. Res., 51, 1-23.

(4) Davis, L. (1987). Genetic Algorithms and Simulated Annealing. Pitman, London, England.

(5) Davis, L. (1991). Handbook of Genetic Algorithms. ed., Van Nostrand Reinhold, New York, New York.

(6) Demmy, W.S. (1979). On the METRIC Distribution Algorithm. Modeling and Simulation, 10, 523-588.

(7) Demmy, W.S. \& Presutti, V.J. (1981). Multi-Echelon Inventory Theory in the Air Force Logistics Command. TIMS Studies in the Management Sciences, 16, 279-297.

(8) Fox, B. \& Landi, M. (1970). Searching for the Multiplier in One-Constraint Optimization Problems. Oper. Res., 18, 253-262.

(9) Goldberg, D.E. (1989). Genetic Algorithms in Search, Optimization, and Machine Learning. Addison Wesley, Reading, Massachusetts. 
(10) Graves, S.C. (1985). A Multi-Echelon Inventory Model for a Reparable Item for one Replinishment. Manag. Sci., 31, 1247-1256.

(11) Holland, J.H. (1975). Adaptation in Natural and Artificial Systems. University of Michigan Press, Ann Arbor, Michigan.

(12) Kim, J.S.; Shin, K.C. \& Park, S.K. (2000). An Optimal Algorithm for Repairable Item Inventory System with Depot Spares. Jour. Oper. Res. Soc., 51, 350-357.

(13) Michalewicz, Z. (1992). Genetic Algorithms + Data Structures = Evolution Programs. Springer-Verlag.

(14) Muckstadt, J.A. (1973). A Model for a Multi-Item, Multi-Echelon, Multi-Indenture Inventory System. Manag. Sci., 20, 472-481.

(15) Muckstadt, J.A. (1978). Some Approximation in Multi-Item, Multi-Echelon Inventory Systems for Recoverable Items. Nav. Res. Logist. Quart., 35, 377-394.

(16) Muckstadt, J.A. \& Thomas, L.J. (1980). Are Multi-Echelon Inventory Methods Worth Implementing in Systems with Low Demand Rate Items? Manag. Sci., 26, 483-494.

(17) Sherbrooke, C.C. (1967). METRIC: Multi-Echelon Technique for Recoverable Item Control. Oper. Res., 16, 122-141.

(18) Syswerda, G. (1989). Uniform Crossover in Genetic Algorithms. Proceedings of the Third International Conference on Genetic Algorithms, San Mateo, California, Morgan Kaufmann Publisher, 2-9

(19) Tedone, M.J. (1989). Reparable Part Management. Interfaces, 19(4), 61-68.

(20) Zorn, W.L.; Deckro, R.F. \& Lehmkuhl, L.J. (1999). Modeling Diminishing Marginal Returns in a Hierarchical Inventory System of Repairable Spare Parts. Ann. Oper. Res., 91, 319-337. 